was continued at a higher temperature for $3 \mathrm{hrs}$. The mixture was cooled to about $100^{\circ}$ and poured into $50 \mathrm{cc}$. of water. To this was added with stirring dil. HCl. The thick, gelatinous, colorless solid that separated was digested for $1 \mathrm{hr}$., collected by filtration, washed with water, and dried. A crude yield of $93 \%$ was obtained. m.p. $84 \sim 85^{\circ}$. Mixed with the keto-acid (26), m.p. $84 \sim 85^{\circ}$, the depression was only $2^{\circ}$.

\title{
Summary
}

$\omega$-Phenyl-fatty acids, $\omega$-phenýl-caproic, -enanthic, -caprylic, -pelargonic, -capric, -undecanoic, -lauric, -tridecanoic, -myristic, -palmitic, -stearic, -arachidic, and -behenic acids, were prepared.

(Received August 20, 1954)

84. Masao Tomita, Yasuo Inubushi, and Kazuo Ito: Studies on the Alkaloids of Menispermaceous Plants. CXIX." A Bisected Phenolic Product from the Cleavage of Tetrandrine and Isotetrandrine with Metallic Sodium in Liquid Ammonia.

(Pharmaceutical Institute, Medical Faculty, University of Kyoto*)

Several years ago, Tomita, Fujita, and Murai reported that on the fission of isotetrandrine $(\mathrm{I}(-,+))^{2)}$ and tetrandrine $(\mathrm{I}(+,+))^{3)}$ with sodium in liquid ammonia, the former yielded as the bisected bases $l-\mathrm{O}, \mathrm{O}, \mathrm{N}$-trimethylcoclaurine (II) and $d-\mathrm{N}$-methylcoclaurine (III), and the latter, $d-\mathrm{O}, \mathrm{O}, \mathrm{N}$-trimethylcoclaurine (II) and $d-\mathrm{N}$-methylcoclaurine (III), respectively; and that $d-\mathrm{N}$-methylcoclaurine (III) thus obtained formed from methanol colorless prisms, m.p. $139 \sim 139.5^{\circ},[\alpha]_{D}^{16}:+88.51^{\circ}\left(\mathrm{CHCl}_{3}\right)$, which by analyses, were found to be a hemihydrate with an empirical formula $\mathrm{C}_{18} \mathrm{H}_{21} \mathrm{O}_{3} \mathrm{~N} \cdot 1 / 2 \mathrm{H}_{2} \mathrm{O}$.

Very recently, however, Kidd and Walker) applied the same mode of fission process to phaeanthine $(\mathrm{I}(-,-))$, an antipode of tetrandrine $(\mathrm{I}(+,+))$, and $\mathrm{O}, \mathrm{O}$-dimethylcurine (IV), and reported that of the two bases (II) and (III) obtained, $l$-N-methylcoclaurine (III), a phenolic base, gave colorless prisms (from benzene or toluene), m.p. 176 177,$[\alpha]_{\mathrm{D}}^{23}$ : $-69.6^{\circ}\left(\mathrm{CHCl}_{3}\right)$, corresponding to a composition of $\mathrm{C}_{18} \mathrm{H}_{21} \mathrm{O}_{3} \mathrm{~N}$.

It is well established that $d l-\mathrm{N}$-methylcoclaurine, when derived from the naturally occurring coclaurine by Tomita and Kusuda, ${ }^{5)}$ as well as when synthesized by Kidd and Walker, ${ }^{4)}$ has a definite m.p. of $161 \sim 162^{\circ}$. However, a doubt has arisen from the fact that of its optically active substances, the $d$-form shows m.p. $139 \sim 139.5^{\circ}$, whereas the $l$-form, m.p. $176 \sim 177^{\circ}$, and hence it has become necessary to reinvestigate the $d$-form (III).

On the other hand, just prior to the above British investigators, Bick and Clezy) also carried out similar cleavage reactions of $\mathrm{O}, \mathrm{O}$-dimethylcurine, $\mathrm{O}, \mathrm{O}$-dimethylchondrocurine, phaeanthine, and isotetrandrine, but they characterized the bisected bases as the methiodides or $\mathrm{O}$-methyl ether methiodides without isolating them per se. As a result, no further records with reference to the m.p. of N-methylcoclaurine (III) of the bisected bases are available.

* Yoshida-Konoe-cho, Sakyo-ku, Kyoto（営时真雄, 犬伏康夫, 伊藤一男).

1) Part CXVIII. Y. Inubushi, M. Kozuka: This Bulletin, 2, 215(1954).

2) M. Tomita, E. Fujita, F. Murai : J. Pharm. Soc. Japan, 71, 226, 1035(1951).

3) E. Fujita, F. Murai : Ibid., 71, 1039(1951).

4) D. A. A. Kidd, J. Walker : J. Chem. Soc., 1954, 669.

5) M. Tomita, F. Kusuda : J. Pharm. Soc. Japan, 72, 280(1952).

6) I I. R. C. Bick, P. S. Clezy : J. Chem. Soc., 1953, 3893. 


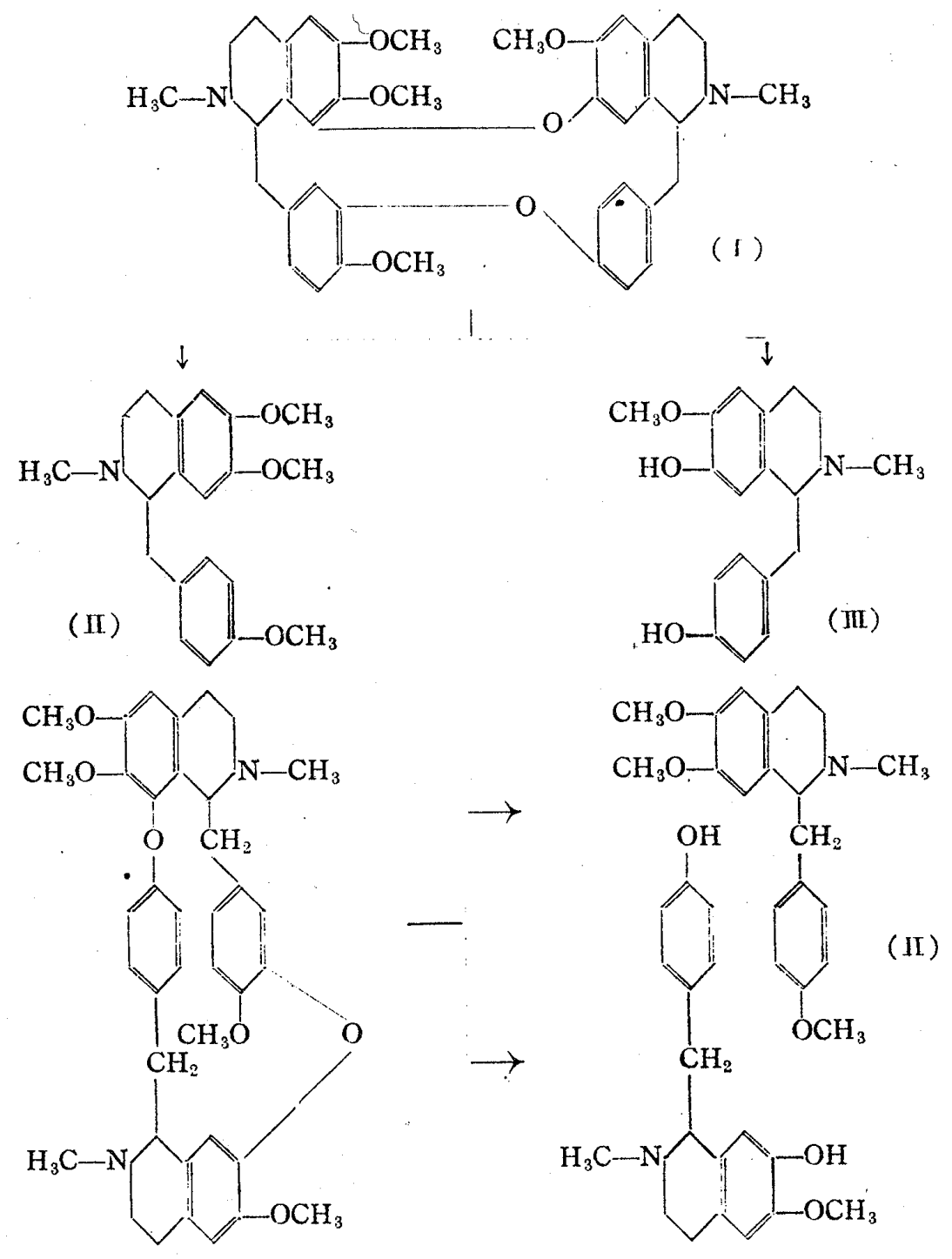

(IV)

(III.)

In view of the fact that the $l$-form (m.p. $176 \sim 177^{\circ}$ ), which crystallized from benzene or toluene, has a molecular formula $\mathrm{C}_{18} \mathrm{H}_{21} \mathrm{O}_{2} \mathrm{~N}$, whereas the $d$-form (m.p. 139 139.5 $)$ which crystallized from methanol, the molecular formula $\mathrm{C}_{18} \mathrm{H}_{21} \mathrm{O}_{3} \mathrm{~N} \cdot 1 / 2 \mathrm{H}_{2} \mathrm{O}$, the first assumption was that the $d$-form having the m.p. of $139 \sim 139.5^{\circ}$ may hold a solvent of crystallization.

When the m.p. of a specimen ${ }^{7}$ ) of the $d$-form (III) obtained by the sodium-liquid ammonia fission of isotetrandrine was again measured, it began to sinter at $140^{\circ}$ and finally melted at $176 \sim 177^{\circ}$. Further recrystallization of this specimen from benzene gave foral aggregates of colorless needles melting at $176 \sim 177^{\circ}$. Contrary to this, the m.p. of a specimen ${ }^{8}$ of the $d$-form (III) obtained by the same mode of cleavage of tetrandrine was again determined and showed m.p. $176 \sim 177^{\circ}$. This phenomenon may be accounted for by the assumption that the substance forming a hemihydrate, m.p. $139 \sim 139.5^{\circ}$, just after preparation, forms an anhydrate with elimination of water of crystallization after preservation for a long period of time. Measurement of the m.p. of specimen ${ }^{9)}$ of the crude substance preserved show about $140^{\circ}$, which after recrystallization from toluene,

7) 'This specimen had been preserved in this Laboratory since 1951 and recorded as m.p. $139 \div 139.5^{\circ}$.

8) This specimen also had been preseved since 1951 and recorded as m.p. $138-139.5^{\circ}$.

9) Recorded m.p. $136 \sim 137^{\circ}$. 
rose to $176 \sim 177^{\circ}$

In order to clarify these situations, we have again taken up an investigation of isotetrandrine by the sodium-liquid ammonia process in the same way as in the previous paper $^{2)}$.. When the crude phenolic base hereby obtained was recrystallized from benzene or toluene, it formed colorless prisms melting at $176 \sim 177^{\circ}$. On the other hand, however,

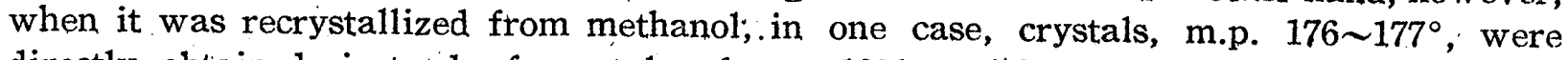
directly obtained, instead of crystals of m.p. $139^{\circ}$, and in another case, prisms of m.p. $138 \sim 140^{\circ}$ were obtained. These facts may be due to the conditions of recrystallization, depending upon the amount of water or moisture contained in the slovent methanol.

The crystals having m.p. $138 \sim 140^{\circ}$ melted at $138 \sim 140^{\circ}$ with some effervescence and on cooling solidified, after which on subsequent heating, they melted at $176 \sim 177^{\circ}$, without sintering at $140^{\circ}$ any longer. When the crystals, m.p. $138 \sim 140^{\circ}$, were further recrystallized from anhydrous ethanol or benzene, they crystallized in colorless prisms melting at $176 \sim$ $177^{\circ}$. Recrystallization of the crystals, m.p. $176 \sim 177^{\circ}$, thus obtained, from methanol or hydrous methanol did not furnish a hemihydrate, m.p. $139^{\circ}$, but a product sintering at $140^{\circ}$ and finally melting at $176 \sim 177^{\circ}$. As a result of the mixed melting point determinations, these crystals showing m.p. $176 \sim 177^{\circ}$ were found to be identical, and to possess analytical values corresponding to a composition $\mathrm{C}_{18} \mathrm{H}_{21} \mathrm{O}_{3} \mathrm{~N}$, having no water of crystallization, $[\alpha]_{\mathrm{D}}^{30}:+62.8^{\circ}$. Furthermore, the paper chromatography of the $d$-form, m.p: $176 \sim$ $177^{\circ}$, and $d l$-form, ${ }^{5)}$ m.p. $161 \sim 162^{\circ}$, revealed that. the two substances have the same Rf value of $0.73 .^{10 \text { ) }}$

The foregoing results are thus summarized as follows : $d-\mathrm{N}-\mathrm{Methylcoclaurine,} \mathrm{when}$ recrystallized from methanol, is obtained as a hemihydrate, m.p. $139 \sim 140^{\circ}$, in some cases, which, after preservation for a long period of time, becomes an anhydrate with elimination of the water of crystallization. On the other hand, however, when it is recrystallized from toluene or benzene instead, it is obtained as m.p. 176 177. , quite identical with that recorded by Kidd and Walker for $l-N$-methylcoclaurine, m.p. $176 \sim 177^{\circ}$.

The authors are indebted to Mr. M. Akasu of Kaken Chem. Drug Co. Ltd., for the generous gift of a sample of isotetrandine. This work was supported by a Grant in Aid for Fundamental Scientific Research from the Ministry of Education, to which the authors' thanks are also due.

\section{Experimental ${ }^{11}$}

Cleavage of Isotetrandrine by Metallie Sodium in Liquid Ammonia-(a) A solution of $2 \mathrm{~g}$. of isotetrandrine in a mixture of $15 \mathrm{cc}$. of benzene and $15 \mathrm{cc}$. of toluene was added dropwise to about $300 \mathrm{cc}$. of liquid $\mathrm{NH}_{3}$ placed in a 1-L. three-necked flask. The temperature was kept at $-35^{\circ}$, and $1.5 \mathrm{~g}$. of $\mathrm{Na}$ was then added in small portions with vigorous stirring until the blue color of the solution persisted for about half an hour, whereafter the mixture of an orange yellow color was kept overnight to permit evaporation of $\mathrm{NH}_{3}$. The residue was treated with $100 \mathrm{cc}$. of water and the resulting solution was extracted with several portions of ether. The ethereal phase was washed with $2 \% \mathrm{NaOH}$ solution and the alkaline washings were combined with the aqueous layer. The etherbenzene-toluene solution was exhaustively extracted with $5 \% \mathrm{HCl}$, and the extract made alkaline with $2 \%$ aq. $\mathrm{NaOH}$. The non-phenolic base was re-extracted with ether, and the ethereal solution was dried over anhyd. $\mathrm{K}_{2} \mathrm{CO}_{3}$ and evaporated, yielding $0.86 \mathrm{~g}$. of an orange red oil.

Meanwhile, the aqueous layer containing the phenolic fraction was neutralized with $\mathrm{HCl}$, made alkaline with $\mathrm{K}_{2} \mathrm{CO}_{3}$, and extracted with ether. The ethereal phase was washed with $2 \% \mathrm{NaOH}$ solution, to which the phenolic base was transferred. The resulting alkaline solution was made acid with $\mathrm{HCI}$ and, after being alkalized with $\mathrm{K}_{2} \mathrm{CO}_{3}$, re-extracted with ether. The ethereal solution was dried over anhyd. $\mathrm{K}_{2} \mathrm{CO}_{3}$ and the ether removed, leaving the crude phenolic base as an orange oil. The oil was dissolved in a small amount of acetone and ether, and the solution allowed to stand,

10) As described in the experimental section, it was carried out according to the method of Kidd and Walker; they also recorded $\mathrm{Rf}$ value of 0.73 for the $l$-form and $d l$-form.

11) All melting points are uncorrected. The microanalyses were carried out in the Microanalytical Laboratory of this Institute by Mr. K. Hozumi and his staff. 
whereupon $0.57 \mathrm{~g}$. of slightly yellowish crystals of m.p. $168 \sim 173^{\circ}$ deposited. Further recrystallization from benzene or toluene yielded floral aggregates of colorless needles, m.p. 176 $177^{\circ} ;[\alpha]_{\mathrm{D}}^{80}:+62.8^{\circ}$ (7.966 mg. Subst. in $1 \mathrm{cc}$. $\mathrm{CHCl}_{3}, l=0.5 \mathrm{dm}$.). Anal. Calcd. for $\mathrm{C}_{18} \mathrm{H}_{21} \mathrm{O}_{3} \mathrm{~N}: \mathrm{C}, 72.21 ; \mathrm{H}, 7.10$. Found : C, $72.26 ; \mathrm{H}, 7.19$.

(b) $2 \mathrm{~g}$. of isotetrandrine dissolved in $20 \mathrm{cc}$. of toluene was cleaved by a procedure similar to that described in the previous paper') and the preducts were separated as above. The yellowiah oily phenolic fraction was dissolved in a small portion of $\mathrm{MeOH}$, and by allowing the solution to stand, $0.6 \mathrm{~g}$. of the crude white crystals were obtained. By recrystallization from $\mathrm{MaOH}$ they crystallized in colorless prisms. A sample, when heated, melted at $138-140^{\circ}$ with frothing, and upon cooling solidified, after which, when again heated, it melted at $176-177^{\circ}$ without melting at around $140^{\circ}$ any longer. Crystallization from abs. EtOH or benzene, however, did not afford the product of m.p. 138 $140^{\circ}$, but of m.p. $176 \sim 177^{\circ}$.

Paper Chromatography - The paper chromatography was carried out according to the method of Kidd and Walker.6) Toyo Roshi No. 50 paper was immersed in $0.2 M$ aq. $\mathrm{KH}_{2} \mathrm{PO}_{4}$, pressed between filter papers to remove excess of the liquid, dried at $100^{\circ}$, and allowed to equilibrate against atmospheric moisture for several hours before use. Development was effected by the descending method with the upper layer of a mixture of $63 \mathrm{cc}$. BuOH, $10 \mathrm{cc}$. AcOH, and $27 \mathrm{cc}$. of water. The Rf values for $d-\mathrm{N}$-methylcoclaurine (m.p. $176 \sim 177^{\circ}$ ) obtained by the above fission, and for $d l-\mathrm{N}$-methylcoclaurine ${ }^{5}$ ) (m.p. $161 \sim 162^{\circ}$ ) (ca. $60 \gamma$ used in each case) were both 0.73 using the Dragendorff reagent.

\section{Summary}

Evidence has been presented that $d$-N-methylcoclaurine (III) forms a hemihydrate having the m.p. of $139 \sim 139.5^{\circ}$, and its anhydrate shows the m.p. $176 \sim 177^{\circ}$, quite identical with that recorded by Kidd and Walker ${ }^{4}$ for $l-\mathrm{N}$-methylcoclaurine, m.p. 176 177 .

(Received August 20, 1954)

85. Masao Tomita and Yoshio Sasaki : Studies on the Alkaloids of Menispermaceous Plants. CXX ${ }^{1)}$. Cleavage of Cepharanthine by Metallic Sodium in. Liquid Ammonia. $(3)^{2}$.

\section{(Pharmaceutical Institute, Medical Faculty, University of Kyoto*)}

In an earlier paperi) of this series, it was shown that as a result of the fission of cepharanthine (I) by the sodium-liquid ammonia process, a base possessing two phenolic hydroxyl and no methoxyl groups, corresponding to $d-1-\left(4^{\prime}-\right.$ hydroxybenzyl $)-6$-hydroxy-Nmethly1-1,2,3,4-tetraydroisoquinoline (II), was obtained in a crystalline form after chromatography, and on this basis formula (I) was proposed for cepharanthine: At that time, however, attempts to isolate the other phenolic fragment in crystalline form, corresponding to $l-1-\left(4^{\prime}-\right.$ methoxybenzyl $)-6$-methoxy-7-hydroxy-N-methyl-1,2,3,4-tetrahydroisoquinoline (III), were unsuccessful. Although the $l$ - and $d$-forms of (III) were obtained previously from $\mathrm{O}$-methyloxyacanthine ${ }^{3)}$ and $\mathrm{O}$-methylrepandine, ${ }^{4)}$ respectively, by the same mode of cleavage, at that time, they were characterized as the corresponding $l$-and $d-\mathrm{O}, \mathrm{O}, \mathrm{N}-$ trimethylcoclaurine (IV), respectively, because of the difficulty of crystallization.

In the present series of experiments, special attention was placed on the base (im) obtainable by the cleavage of cepharanthine, and this paper deals with the isolation of this particular base (III) from the resinous phenolic fraction left after the removal of the base (II). As described in the experimental section, a product forming a crystalline

* Yoshida-konoe-cho, Sakyo-ku, kyoto（篦田寡雄，佐々木喜男).

1) Part CXIX. M. Tomita, Y. Inubushi, K. Ito: This Bulletin, 2, 372(1954).

2) Part (2). M. Tomita, Y. Sasaki : Ibid., 2, 89(1954).

3) E. Fujita : J. Pharm. Soc. Japan, 72, 213, 217(1952).

4) E. Fujita, T. Saijoh : Ibid., 72, 1232(1952). 